International Journal of Advanced Technology in Mechanical, Mechatronics and Materials
(IJATEC)
Vol. 02, No. 1 (2021) $1-10$
Institute for Research on Innovation and Industrial System (IRIS)

\title{
Design Color Sorting with Conveyor and Robot Arm Using Lego Mindstorms NXT
}

\author{
Yudhi Gunardi ${ }^{*}$, Danang Fajar Kuncoro ${ }^{\mathrm{a}}$, Jumadril JN ${ }^{\mathrm{b}}$ \\ ${ }^{a}$ Department of Electrical Engineering, Faculty of Engineering, Universitas Mercu Buana, Jakarta, Indonesia \\ bUniversitas Batam, Batam, Indonesia
}

\begin{abstract}
Lego Mindstorms education robot is an interactive robot because it can be used to design various kinds of robot applications. This robot can be used to design sorting robots before they are applied to real robots. Many sorting techniques are in the form of colorful objects, but only for onetime sorting. This research will design and implement a color sorting robot with 2 detection stages, namely the conveyor and the robot arm, the initial sorting is carried out by the conveyor for sorting color objects, the next sorting uses the robot arm. to move the selected color object to a place that matches the desired color. The results obtained in sorting color objects using 1 color sensor and Bluetooth for communication between the conveyor and the robot arm via Bluetooth works well where three objects in red, blue and green can be moved according to the desired color object.
\end{abstract}

Keywords: color sorting; robot arm; conveyor; Lego Mindstorms

DOI: $10.37869 /$ ijatec.v2i1.30

Received 31 August 2020; Accepted 2 April 2021; Available online 19 April 2021

(C) The Authors. Published by IRIS. This is an open access article under the CC BY-NC-SA license cc) (1) (2)(2).

\section{Introduction}

The development of robot technology is very rapid, marked by advances in robot intelligence, robot intelligence is determined by the robot's ability to work optimally. Robot intelligence supporting tools in the form of sensors and actuators. Sensors are part of the robot that functions to receive information signals from the environment and forward them to the processor. Meanwhile, the actuator is the part of the robot that is used to perform the action ordered by the control unit.

One of the industrial applications is a color object sorting robot, many workers who when sorting will feel tired, causing errors in sorting objects, for this reason, robot technology can help humans to work continuously without experiencing errors.

Currently, a lot of research is being done, especially in the field of sorting objects by color, arm robot control using genetic algorithm methods used for logistics and scheduling [1], robot control simulation and dual arm design using MATLAB simulation [2], arm robot control using image algorithms used for logistics and scheduling [3], arm robot control using MATLAB simulation [4], robot arm control using voice [5], focused in one color object sorting process [6], simulation robot [7-8].

LEGO Mindstorms' education robot is an interactive robot because it can be used to design various kinds of robot applications such as Nxt robot design for robot movement with front-wheel rotation of bicycle tires [9], telling the development technology of the robot nxt [10], Designing the Lego nxt robot movement using fuzzy method for wheeled robot [11].

Therefore, the Lego Mindstorms robot is an interactive robot because it can be used to design various kinds of robot applications, in this research the Lego robot is used to design a sorting robot 
with 2 detection stages, namely the conveyor and the robot arm, initial sorting is carried out by the conveyor for sorting color objects. , the next sorting uses the robot arm to move the object of the selected color to a place that matches the desired color by using 1 color sensor that is shared by the conveyor and the robot arm where the sensor will provide information to the conveyor and the robot arm using Bluetooth.

\subsection{Color theory}

Color can be defined as part of the addressing of the sense of vision, or as the nature of the light emitted. The process of visible color is due to the presence of light that overrides an object, and it reflects light to our eye (retina) until the color is visible. Objects are red because the pigment properties of these objects reflect red and absorb other colors. Objects are black because the pigment properties of the object absorb all colors. On the contrary, an object is white because the pigment properties of the object reflect all colors. Theories and color recognition have been widely presented by experts, including Young and Helmholtz's discovery proves that there is a link between the color of light coming to the eye and the color received in the brain.

This was an early endorsement of Newton's assumptions about light and the colors of objects. Newton's assumption states that the actual colored objects are mere receivers, absorbers, and successors to the color of light that exist in the spectrum. James Clerk Maxwell created a series of experiments using light projectors and color filters. Three projectors that have been filtered with different colors are highlighted to a white screen in a dark room.

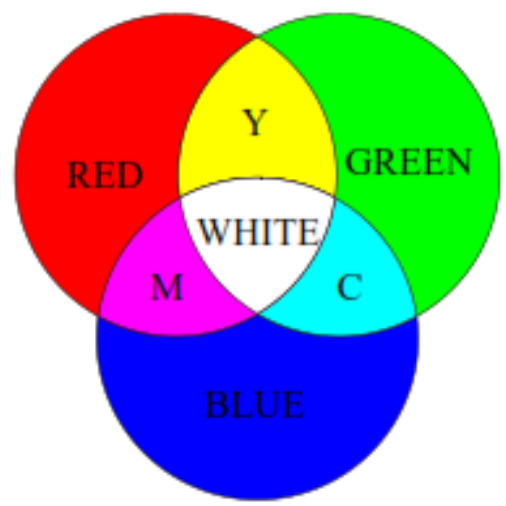

Figure 1. Maxwell primary color experiment diagram image

The stacking of two or three colored light turns out to produce another light color (unknown) in mixing colors using ink/paint/dye. Stacking (mixing) green light and red light, for example produces yellow color. Maxwell's experiments concluded that green, red and blue are the primary colors in mixing light colors. Primary colors are colors that cannot be produced through mixing any color. Through these primary colors of light (blue, green, and red) all the colors of light can be formed and created. If these three primary light colors in maximum intensity are combined, based on the experiments of 3 projectors that Maxwell demonstrated. Maxwell's experiments are excellent models or imitations to facilitate our understanding of how eye receptors capture light resulting in colored vision in the brain.

\subsection{Robot LEGO Mindstorms NXT 2.0}

LEGO Mindstorms NXT is an educational robot device from LEGO. The NXT series was officially released in 2006 as the successor to the previous series, RIS (Robotics Invention System) which was successful in the market. The use of Mindstorms NXT helps make it easier to create robots. This is because Mindstorms NXT eliminates the need to solder circuits and eliminates difficulties when installing motors. Robots made using LEGO cannot be considered a final robot but can be considered a model. LEGO robot devices are available in 2 versions, retail and educational. LEGO Mindstorms NXT has several main components such as processors and sensors.

\subsection{NXT Brick}


NXT Brick is the brain and power source of the LEGO Mindstorms NXT robot. The program that has been created will be entered and run by NXT Brick. The robot can receive information such as touch, sound, or light intensity according to the sensor used. The information is sent to NXT Brick for further processing. NXT Brick also provides the power needed for the motor to move.

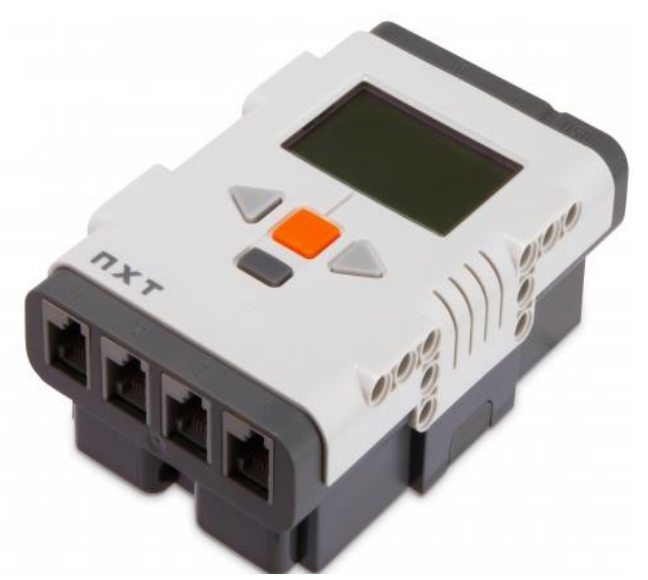

Figure 2. Lego Mindstorms NXT intelligent

Features of NXT Brick include the following:

a. Four sensor ports: 1 to 4

b. Three motor ports: A, B, and C.

c. USB port to receive and transmit data.

d. Bluetooth that can be used to receive and/or transmit data.

e. FLASH $256 \mathrm{~KB}, \mathrm{RAM} 64 \mathrm{~KB}$

f. $100 \times 64$ pixel LCD.

g. AA battery as a power source

\subsection{Color Sensor}

The robot also needs inputs that will determine what the robot should do. This input generally enters the robot's brain in a variety of ways. Some use the remote or are given before the robot is activated. And there are also those directly given to robots through its program. In this third type, once the robot is turned on it will run what is already determined for him. This is very true for industrial robots in general. Meanwhile, for the first and second types are somewhat different. Orders are coming from the outside. Of course, these commands must be in accordance with the ability of the robot itself, because otherwise, of course the robot will not run it. Robots can also receive input from the robot itself without direct human intervention, i.e., through sensors. The color sensor is one of the sensors that gives the robot vision (like an ultrasonic sensor). Color sensors have three different functions in one tool.

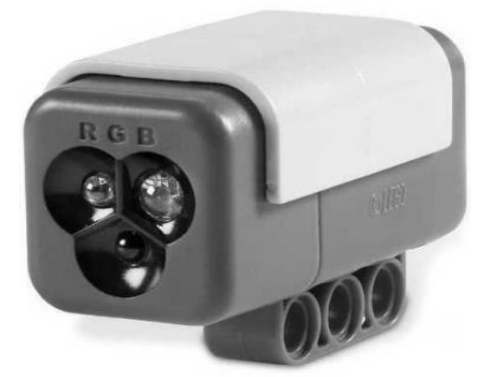

Figure 3. RGB color sensor Lego NXT

Color sensors allow robots to distinguish between color, light and dark. The sensor can detect 6 different colors, read the intensity of light indoors and measure the light intensity of colored surfaces. 
Color sensors can also be used as color lights. In using this color sensor as a sensor, there are 3 main functions that must be considered:

a. Use the sensor as a color sensor

To get optimal color detection, the sensor must be directed at an exact angle about $1 \mathrm{~cm}$ to the surface. Incorrect color readings may occur if this sensor is directed at another angle to the surface or if used in bright light.

b. Using sensors as light sensors

Sensors can be used to pick up single light intensity readings. It serves as a light sensor when the light color is set to red. Using light colors (green or blue) can give different results. This sensor can be used to read light intensity from the environment or reflected light. One of the three colors can shine when reading reflected light.

c. Using sensors as color lamps

We can use sensors as color lights to control individual output colors (red, green, or blue) and add personality to robots.

In physics, colors are composed of basic colors. For light, the basic color of the constituent is red, green and blue, or better known as RGB (Red-Green-Blue). The color parameters have different light waves. Light is actually an electromagnetic wave that comes with different wavelengths.

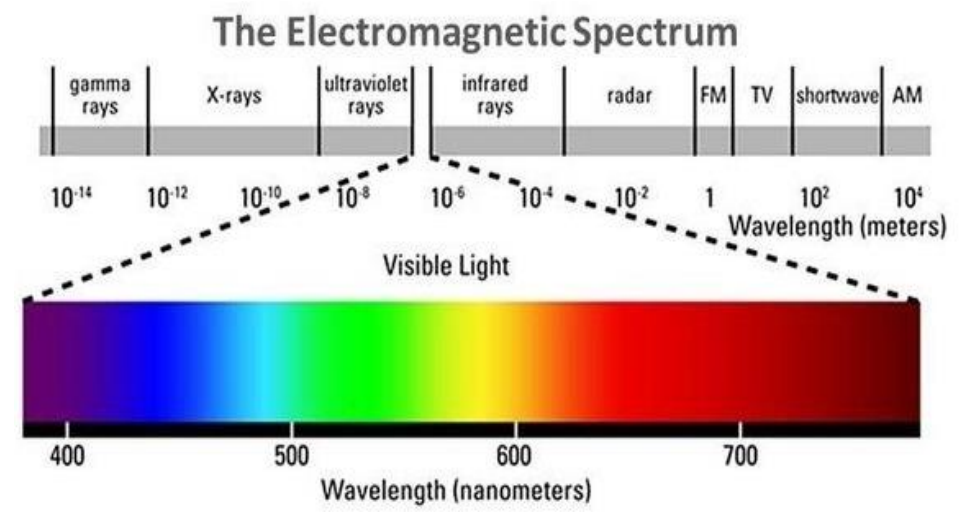

Figure 4. Wavelength color

This wavelength enters the eye, and its energy is absorbed by the cell at the back of the eye. This light energy is then converted into chemical energy which is then processed by several hundred million special neurons and after one a few seconds, the brain tells it that the wavelength of $450 \mathrm{~nm}$ is blue and the wavelength of 520 is green. And at the conscious level, it is never really looking at those wavelengths, but it's just the result of what the brain knows. Through this process, too, many other wavelengths are rejected by the brain, as are infrared and ultraviolet. These wavelengths are there too, but we can't see them.

\subsection{Servo Motor}

Three servo motors give the robot the ability to move. If using moving blocks in Mindstorms NXT-G software for motor programs, two automatic motors will synchronize, so the robot will move in a straight line. Figure 5. The built-in rotation sensor in each motor also allows to set different speeds for the motor by setting different power parameters in the software.

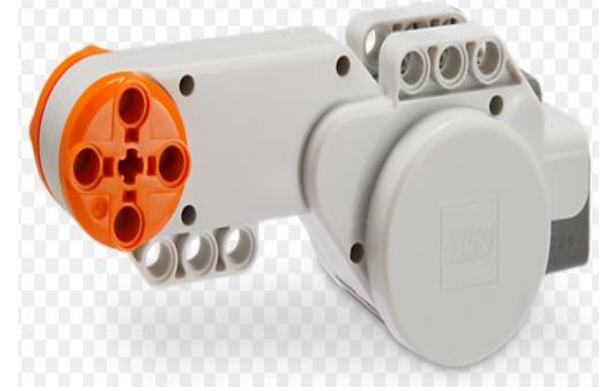

Figure 5. Lego Servo Motor 
Servo Motor Each motor has a built-in rotation sensor. This makes it possible to control the robot's movements precisely. The sensor measures the rotation of the motor in degrees or full rotation \pm \pm 1 degree). One rotation equals 360 degrees, so if you set the motor to rotate 180 degrees, the output shaft will make half the rotation.

\subsection{Software NXT-G}

NXT-G software is an important factor in the design stage of the robot. The software is a motion algorithm and robot task in the form of program listings embedded into microcontrollers. The program can be various versions and programming languages, according to the specifications of the microcontroller used.

The NXT-G program is software that will be used to tell robots what to do. NXT-G makes it possible to create unloadable programs for Lego Mindstorms robots [12]. These programs can be simple instructions such as "move forward or stop within $2 \mathrm{~cm}$ ". Lego Mindstorms robots can be built with a variety of motors and sensors. But without a good program, robots will not know what to do.

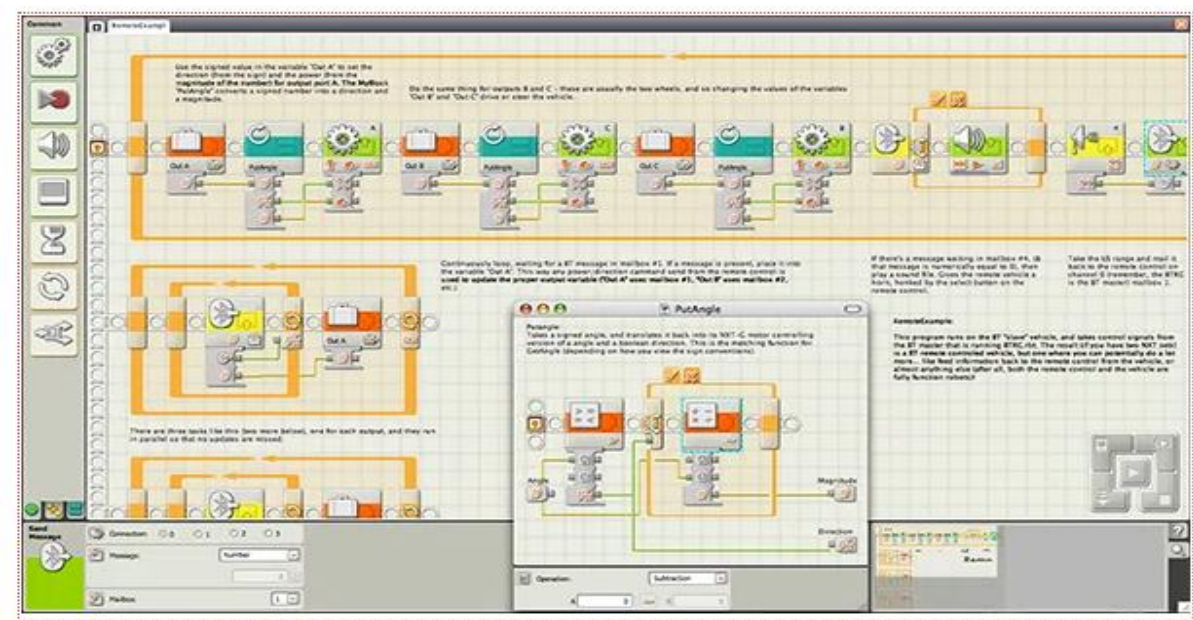

Figure 6. The NXT-G program

Workspace program of Lego Mindstorms software NXT-G, very interesting because all the commands for the program are already available, we just take the command through the image to do the robot work.

\section{Working Principle}

The working principle of the robot that I made is the Lego Mindstorms NXT robot that uses a conveyor and arm robot work system. For how the conveyor works, it functions to carry boxes and sort them with a color sensor to separate the red, green and blue boxes. Then after the box is separated and enters the container according to the predetermined color, then the arm robot works by transporting and moving the box to the final container that also matches the color of the box.

From the flowchart above can be explained there are 3 objects in red, green and blue that will be done separation from each object. The first separation is done when the object comes through the conveyor that sorts through the color sensor against the object by color, when it detects the color via Bluetooth the result of separation on the conveyor will be informed to the robot arm about the colored object, upon arrival the object will move the object to a place corresponding to the color that comes.

There are 3 main controls in this circuit:

1. Color sensor RGB

2. DC motor

3. Bluetooth 


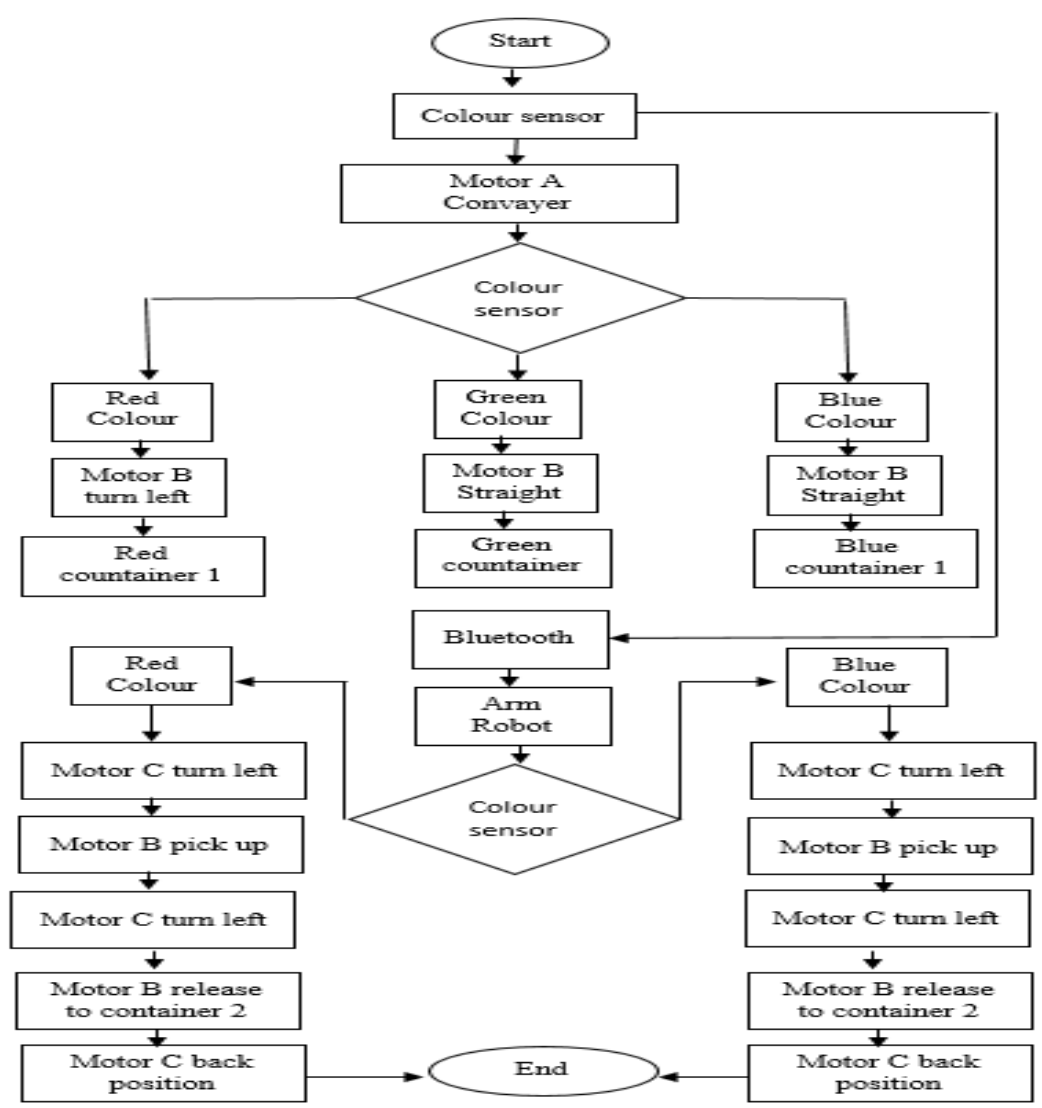

Figure 7. flowchart of how the conveyor robot $\&$ arm robot works

\subsection{Color sensor $R G B$}

RGB color sensor on Lego Mindstorms usually given icon as in Figure 8, where this symbol is an icon for color sensor.

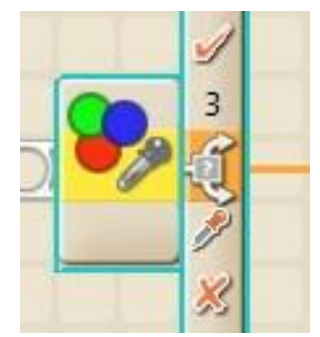

Figure 8. Color sensor icon

We can detect the color of objects that can be detected by those color sensors. The symbol above with the question mark is an icon branching, which serves to determine the action of a program if the given condition is met or not, the ticker symbol is when the conditions we provide are met. And the cross sign symbol states if the conditions we provide are not met.

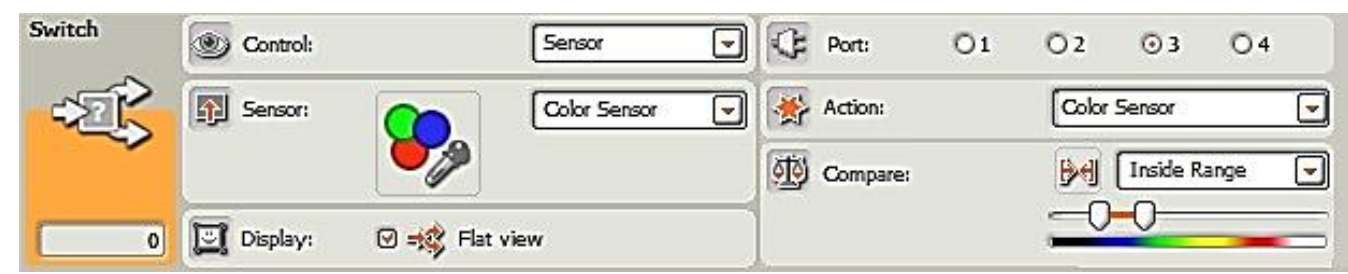

Figure 9. RGB sensor detects blue color

There are 6 ranges of detectable colors are black, blue, green, yellow, red and white. In this icon is used an icon that is color sensor. In the parameters above set to give the specified command, if detected a certain color. In this program set up a program to detect the colors blue, green and red. 


\subsection{Motor}

Figure 10 is an icon for motor drive. The letter $\mathrm{C}$ on the upper right corner states that the $\mathrm{C}$ motor is moving.

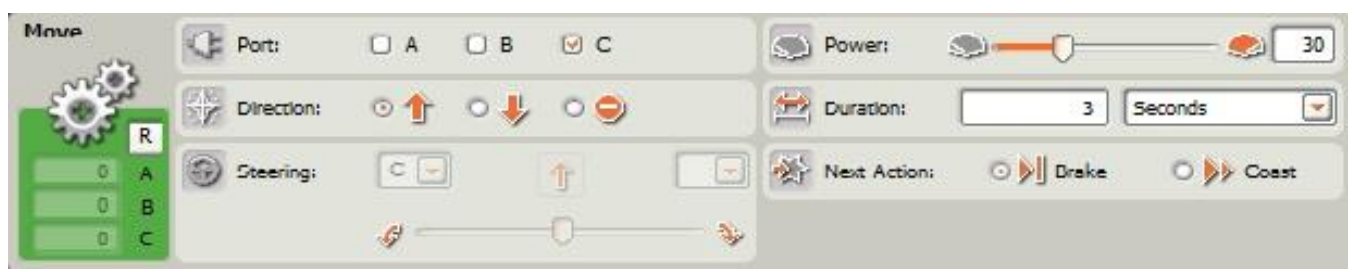

Figure 10. motor movement

The arrow on the lower left corner indicates the direction of movement of the motor. The symbol in the middle is a symbol for the power motor by $50 \%$ within 3 seconds or the speed of the motor. And the symbol on the lower right corner states that the length of the motor moves in seconds.

\subsection{Bluetooth}

In figure 11 are icons and parameters to set give commands via Bluetooth specified, if detected a specific color.

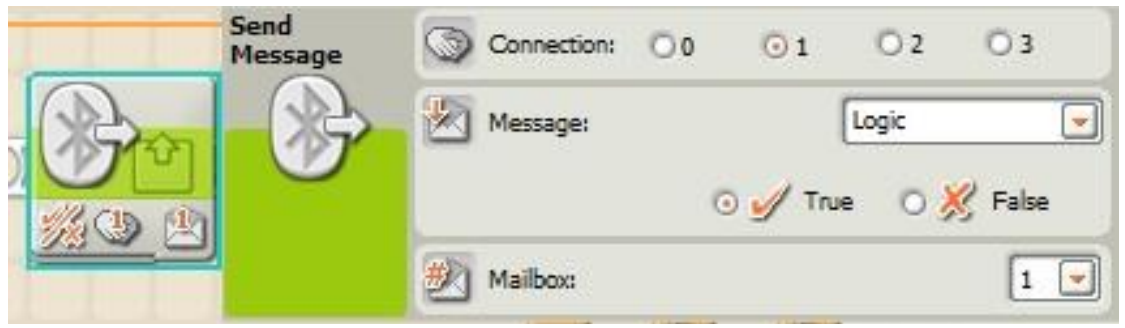

Figure 11. Bluetooth

The above Bluetooth icons and parameters serve to send data from the conveyor to the robot arm according to the mailbox, where mailbox 1 is set to red.

\subsection{Conveyor Programming}

NXT-G program. Where the program uses the icons that have been provided, we only need to arrange them according to the program we want. We also usually use mathematical logic in compiling the program.

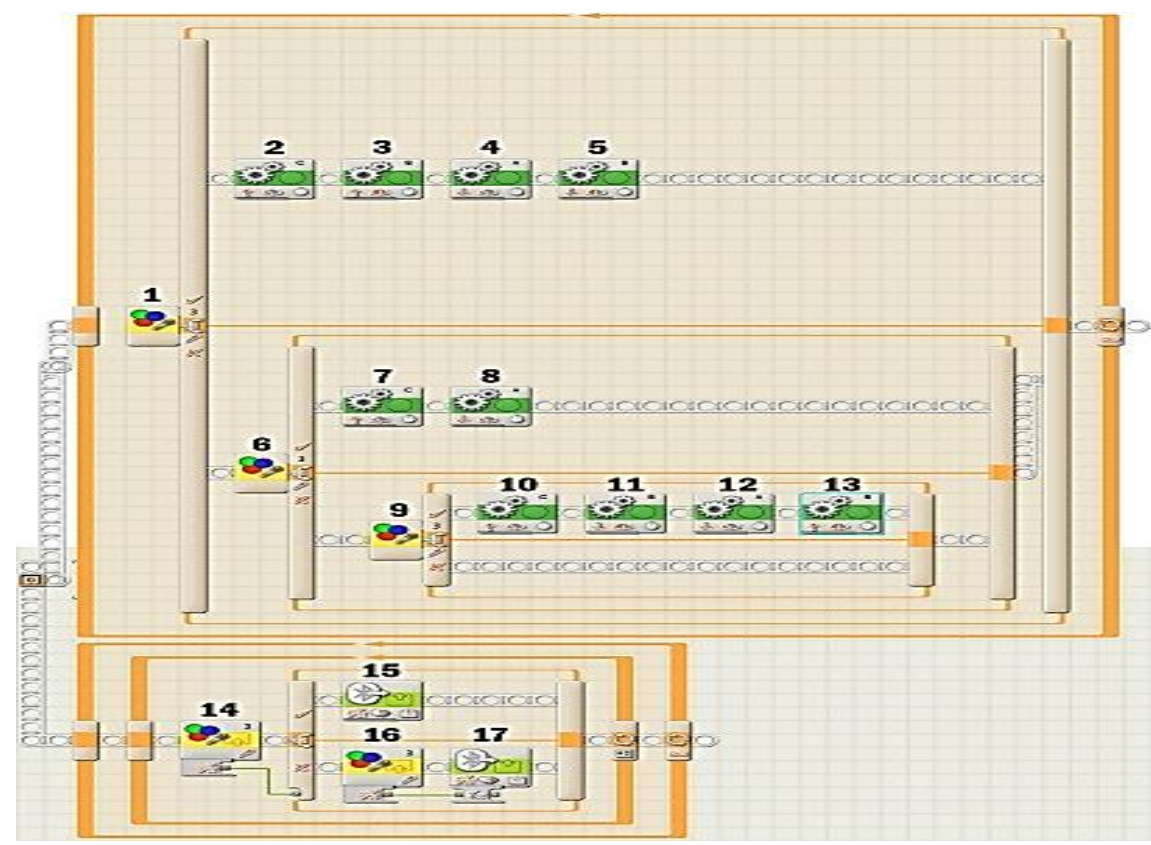

Figure 12. Conveyor robot program 
Numbers 1, 6, 9, 14, 16 are color sensors, numbers 15 and 17 are bluetooth, while numbers 2, 3, 4, 5, $7,8,10,11,12,13$ are the motor programming process at the converter up to 13 are programs DC motor used for conveyor movement.

\subsection{Robot Arm Programming}

The movement of the robot arm begins when the robot arm gets a Bluetooth signal from the conveyor.

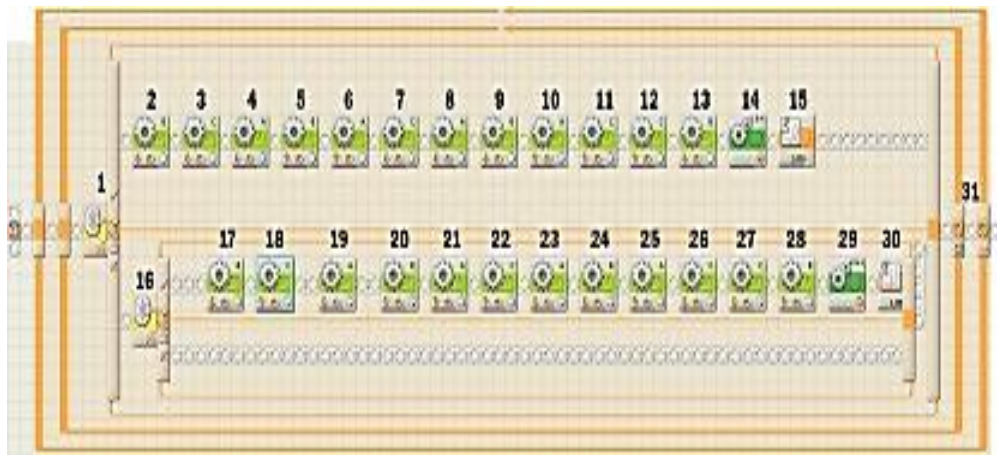

Figure 13. Robot Arm Program

Number 1, 16 is Bluetooth, and number 2 - 30 is the setting of the motor robot arm motion

\section{Results and Discussions}

For the simulation arena, an activity map is made as follows:

a. There are 3 places for goods consisting of 3 baskets in blue, green and red

b. Item transfer occurs when the item is red and blue which will be moved to another location by the robot arm according to the color of the item.

c. Green items are not moved.
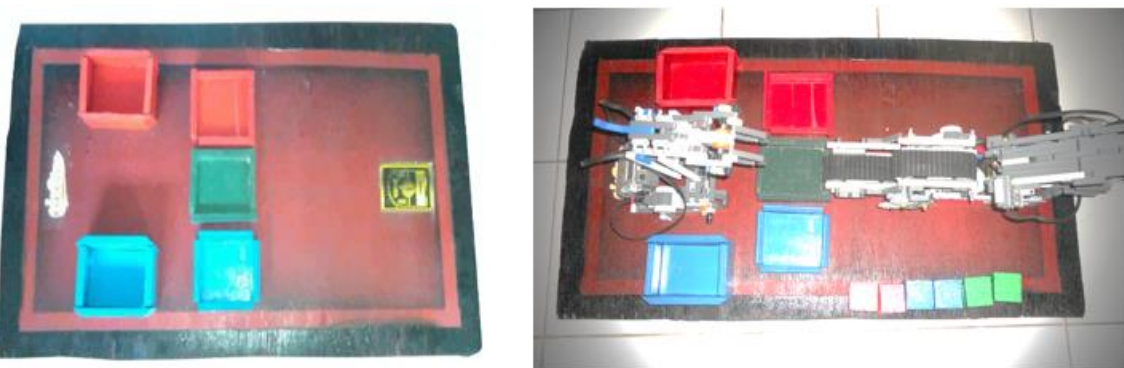

Figure 14. Design arena
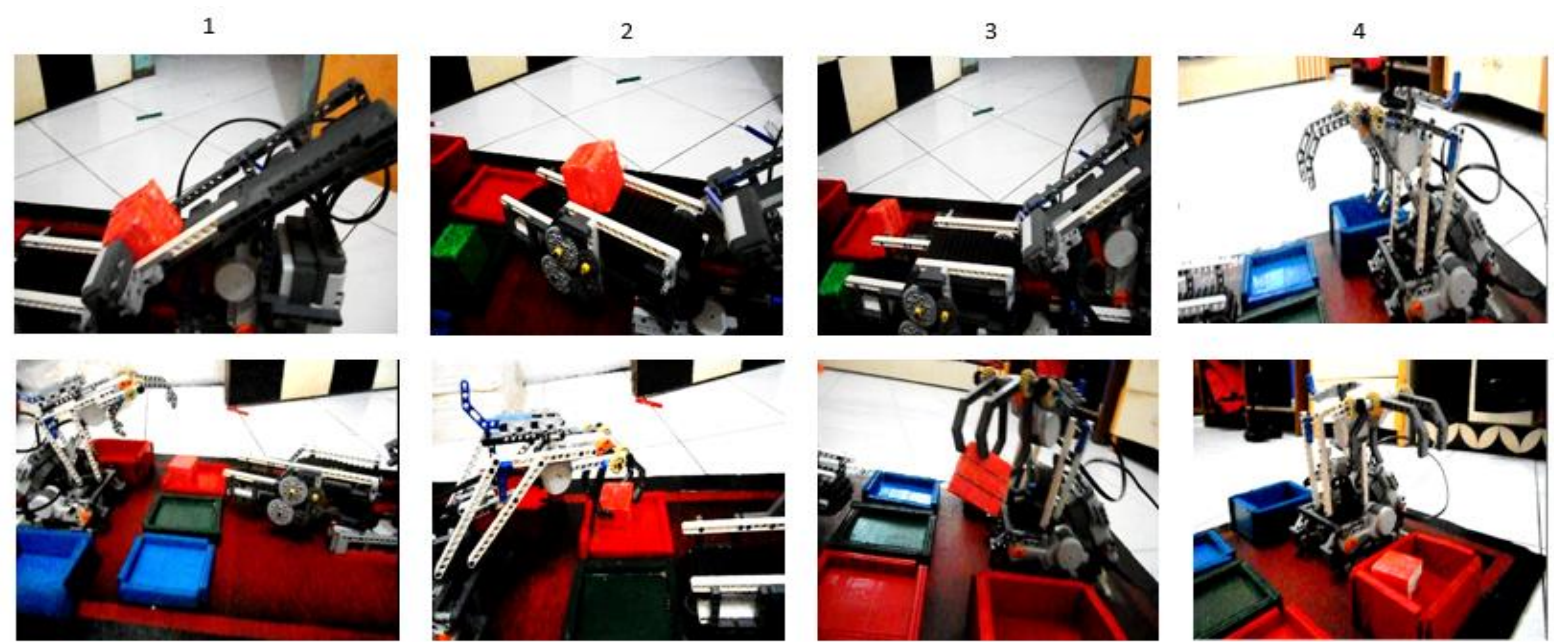

Figure 15. The red sorting process on a conveyor 
In the above process the conveyor will move to the red 1 container after receiving instructions from the color sensor, when the color detection is successful, the color sensor will send information via Bluetooth to the robot arm to go to red to pick up a red object and move it to the container 2 . The same process When the blue object occurs, the blue object is moved from container 1 to container 2 . On the green object, the transfer occurs only on counter 1 .

Table 1. Conveyor motion

\begin{tabular}{ccc}
\hline & \multicolumn{2}{c}{ Conveyor } \\
\hline Colour & Sign & Information \\
red & $\sqrt{ }$ & Succes to container 1 \\
green & $\sqrt{ }$ & Succes to container 2 \\
blue & $\sqrt{ }$ & Succes to container 3 \\
\hline
\end{tabular}

Table 2. Arm robot motion

\begin{tabular}{ccc}
\hline & \multicolumn{2}{c}{ Arm Robot } \\
\hline Colour & Sign & Information \\
red & $\sqrt{ }$ & Succes to container 2 \\
blue & $\sqrt{ }$ & Succes to container 3 \\
\hline
\end{tabular}

Table 3. Sensor Color

\begin{tabular}{ccc}
\hline & \multicolumn{2}{c}{ Sensor Color } \\
\hline Colour & Sign & Information \\
red & $\sqrt{ }$ & detect \\
green & $\sqrt{ }$ & detect \\
blue & $\sqrt{ }$ & detect \\
\hline
\end{tabular}

Table 4. Communication Sensor Colour + bluetooth

\begin{tabular}{ccc}
\hline \multicolumn{3}{c}{ Sensor Colour + bluetooth } \\
\hline Colour & Sign & Information \\
red & $\sqrt{ }$ & detect \\
green & $\sqrt{ }$ & detect \\
blue & $\sqrt{ }$ & detect \\
\hline
\end{tabular}

\section{Conclusion}

Based on the results of testing and analysis that has been described can be concluded, namely:

a. From the sensor testing used, it can be concluded that the sensor used for this Lego robot works well.

b. Like a color sensor that can detect colors and sort them. Like a conveyor robot that detect red, green and blue to sort the boxes and bring the boxes to the starting container.

c. The robotic arm can also move the red and blue boxes from the initial container to the final container.

d. Communication between conveyor and robot arm can run well through Bluetooth technology.

\section{References}

[1] S. Harun and M. F. Ibrahim, "A genetic algorithm based task scheduling system for logistics service robots," Bull. Electr. Eng. Informatics, vol. 8, no. 1, pp. 206-213, 2019.

[2] B. Liu, "Design and Analysis of Dual-arm SCARA Robot Based on Stereo Simulation and 3D Modeling," 2018 IEEE Int. Conf. Inf. Autom., no. August, pp. 1233-1237, 2018.

[3] K. Hwang, J. Lee, A. I. Base, and V. Servoing, "Intelligent Image Base Visual Servoing controller for Robot Arm," IEEE Conf. Intell. Transp. Syst. Proceedings, ITSC, pp. 33-34, 2017.

[4] K. A. Gouda and K. A. Bozed, "Kinematics Analysis and Simulation of a Robotic Arm using MATLAB 3 2," in Proceedings 2016 4th International Connce on Control Engineering \& Information 
Technology (CEIT -2016) 16-18 December 2016 - Hammamet, Tunisia Kinematics, 2016, no. December, pp. 16-18.

[5] C. Wang, "Voice control dual arm robot based on ROS system," in Proceedings of the 2018 IEEE International Conference on Intelligence and Safety for Robotics Shenyang, China, August 24-27, 2018, 2018, pp. 232-237.

[6] C. Budaciu and L. Apostol, "Dynamic Analysis and Control of Lego Mindstormss NXT Bicycle," in 201620 th International Conference on System Theory, Control and Computing (ICSTCC), October 1315, Sinaia, Romania Dyn, 2016, pp. 145-149.

[7] Adriansyah. Andi, Sulle. B, Ihsanto. E, Gunardi. Y, "Optimization of circular robot size using behavior based architecture", journal of Telecommunication, Electronic and Computer Engineering (JTEC), vol. 9, pp. 67-72,2017

[8] Supegina Fina, Manalor Indrianus, Gunardi Yudhi. Perancangan Telerobotik Sederhana Dengan Kamera WiFi. Urnal Jurusan Teknik Elektro, Fakultas Teknik, Universitas Mercu Buana, Vol. 2, No. 2, Jakarta Juni 2016.

[9] K. Poikselk, "Evolutionary Robotics on Lego NXT Platform," in lligence 2015 IEEE 27th International Conference on Tools with Artificial Intelligence Evolutionary, 2015.

[10] N. F. J. and N. M. A. G. M. A. AKMAL, "fuzzy logic controller for two wheeled ev3 Lego robot," IEEE Conf. Syst. Process Control (ICSPC 2017), no. December, pp. 15-17, 2017.

[11] C. Lapusan, O. Hancu, C. Rad, L. Dache, and V. Maties, "Integrated learning platform based on Lego NXT and Matlab for teaching mechatronics," in ECAI 2016 - International Conference - 8th Edition Electronics, Computers and Artificial Intelligence 30 June -02 July, 2016, Ploiesti, ROMÂNIA, 2016.

[12] Jatmiko, W. et al. 2010. RobotLego Mindstormss: Teori danPraktek. Jawa Barat : Fakultas Ilmu Komputer universitas Indonesia. 